\title{
The Psychological and Sleep-Related Impact of Coronavirus Disease 2019 (COVID-19): A Systematic Review
}

\author{
Tetti Solehati ${ }^{1}$, Cecep Eli Kosasih ${ }^{2 *}$, Yanti Hermayanti ${ }^{1}$, Henny Suzana Mediani ${ }^{3}$
}

\author{
${ }^{1}$ Department of Maternity, Faculty of Nursing, Padjadjaran University, West Java, Indonesia \\ ${ }^{2}$ Department of Critical Care and Emergency Nursing, Faculty of Nursing, Padjadjaran University, West Java, Indonesia \\ ${ }^{3}$ Department of Pediatric, Faculty of Nursing, Padjadjaran University, West Java, Indonesia
}

\begin{abstract}
The coronavirus disease 2019 (COVID-19) pandemic has had a global impact on mental health and sleeps quality that is unprecedented in the 21 st century. This systematic review aimed to assess the psychological impact of COVID-19 and its effect on people's sleep. Systematic searches were conducted via PubMed, ScienceDirect, and ProQuest from 2019 to August 2020, following PRISMA guidelines. Articles were selected based on eligibility criteria such as having a cross-sectional study design, assessing the general public's mental health status or sleep problems, medical workers and non-medical workers during the COVID-19 pandemic, and using standardized and validated scales for measurement. The keywords: COVID-19 OR SARS-CoV-2 AND Mental health OR Psychological health AND General population OR General public AND sleep AND Impact of Coronavirus disease 2019. A total of 23 articles were selected after being assessed. During the period of the COVID-19 pandemic, medical workers were found to have psychological problems such as anxiety, depression, stress, Post-Traumatic Stress Disorder (PTSD), psychological distress, somatization symptoms, suicidal ideation, high risk of severe mental illness, worry, and insomnia. Non-medical workers experienced symptoms of depression. The general public experienced psychological problems: depression, anxiety, stress, bad mood, inability to stop thinking about COVID-19, panic, and experiencing sleep problems such as changing sleep patterns, sleep disturbances, sleep quality, difficulty with sleep initiation, and shortened sleep duration. The COVID-19 pandemic has been associated with psychological problems and sleep disorders for medical workers, non-medical workers, and the general public.
\end{abstract}

Keywords: COVID-19, medical workers, mental health, psychological impact, the non-medical

\section{Introduction}

The viral outbreak of coronavirus disease 2019 (COVID-19) has become a major global issue and the most severe threat to public health worldwide in the 21 st century. ${ }^{1}$ The disease was named by the World Health Organization (WHO) as coronavirus disease 2019 (COVID-19) in February 2020. ${ }^{2}$ The disease reportedly started in Wuhan, China, in December 2019, ${ }^{3}$ and spread to other countries. ${ }^{4}$ On March 11, 2020, WHO declared this virus outbreak as a global pandemic spreading rapidly, and more than 20,000,000 cases have occurred globally. ${ }^{2}$

The COVID-19 disease has caused many deaths. As of February 11, 2020, a total of 44,672 reported cases of COVID-19 in Henan Province, China, including 1,716 among health workers, resulting in 1,023 deaths, including at least five among health workers. ${ }^{5}$ According to WHO, 6,040,609 confirmed cases and 370,657 deaths had been reported globally as of June $1,2020 .{ }^{6}$ The COVID-19 epidemic has become a life-threatening pandemic that causes many deaths quickly.

Correspondence*: Cecep Eli Kosasih, Department of Critical Care and Emergency Nursing, Faculty of Nursing Padjadjaran University, Indonesia, E-mail: cecep.e.kosasih@unpad.ac.id, Phone: +62 81320941727
Pandemics cause drastic changes to the lives of people in global, public, and private economic aspects. ${ }^{3}$ To control the spread of COVID-19, quarantine and lockdown were carried out. ${ }^{7}$ This situation causes economic recession and unemployment. COVID-19 has also had psychological consequences. ${ }^{1,3}$ The viral outbreak created uncertainty and fear, which can lead to an increase in suicide as well as mental disorders associated with suicide. ${ }^{3}$ In the US, France, India, Pakistan, Italy, and Germany, there was an increase in the trajectory of suicides due to the pandemic. ${ }^{8}$ The study of Thakur and Jain has reported that there has been increased psychological distress among the general population during this pandemic, people with pre-existing mental disorders, and healthcare workers. ${ }^{8}$

Literature review studies on the general population in China, Spain, Italy, Iran, the US, Turkey, Nepal, and Denmark showed that levels of anxiety symptoms during the COVID-19 pandemic are relatively high $(6.33 \%-$ $50.9 \%)$, as are levels of depression $(14.6 \%-48.3 \%)$, post-traumatic stress disorder $(7 \%-53.8 \%)$, psycholo-

Received : April 16, 202

Accepted : June 15, 2021

Published: July 31, 2021 
gical stress $(34.43 \%-38 \%)$, and stress $(8.1 \%-$ $81.9 \%){ }^{3}$ In addition to the general public, COVID-19 also has a psychological impact on health workers. They are directly involved in the treatment and care of COVID-19 patients. ${ }^{9}$ Healthcare workers who work in a SARS unit, or have family and friends infected with SARS, have much more significant psychological problems than those who do not have similar experiences. 10 The COVID-19 pandemic has led to increasing psychological problems that can disrupt lives.

The COVID-19 outbreak has caused sleep problems among the general public, medical workers, and non-medical workers. Sleep problems have also occurred among healthcare workers. ${ }^{11}$ To combat COVID-19, health workers have been burdened substantially and have had their sleep patterns disturbed. Sleep problems can affect the body's immune status and mental health, quality of life and productivity of a person, may have negative implications on health, quality of work and increase the risk of adverse events and behavior that endanger safety, as well as the risk of work-related accidents or injuries. ${ }^{12}$ The COVID-19 pandemic has caused unprecedented mental health hazards as well as sleep problems globally. ${ }^{3}$ Serious attention must be given to the public's mental health and sleep needs in general, including medical workers and non-medical workers. Appropriate policies are needed to overcome these problems.

Although there is a great deal of literature on psychological impacts, little information is available regarding the psychological and sleep-related effects on the general public, medical workers, and non-medical workers during the COVID-19 outbreak. Therefore, a review was needed that will reveal the impact systematically. This systematic review aimed to assess the psychological impact of COVID-19 and its effect on people's sleep for the general public, medical, and non-medical workers.

\section{Method \\ Search Strategy}

This study used the PICO (Population, Interest, Comparison, and Outcome) approach to develop keywords. Population: general public, medical workers, and non-medical workers. Interest: impact of COVID-19. Comparison: -. Outcome: mental health, sleep. The met-hod and results of this systematic review were based on PRISMA (Figure 1). ${ }^{13}$ Systematic searches were conducted via PubMed, ScienceDirect, ProQuest from 2019 to August 2020 following PRISMA guidelines (Figure 1). The search terms used were: (COVID-19 OR SARSCoV-2) AND (Mental health OR Psychological health) AND (General population OR General public) AND Medical workers AND Non-medical AND sleep AND Impact of Coronavirus Disease-2019 using three databases by using the same

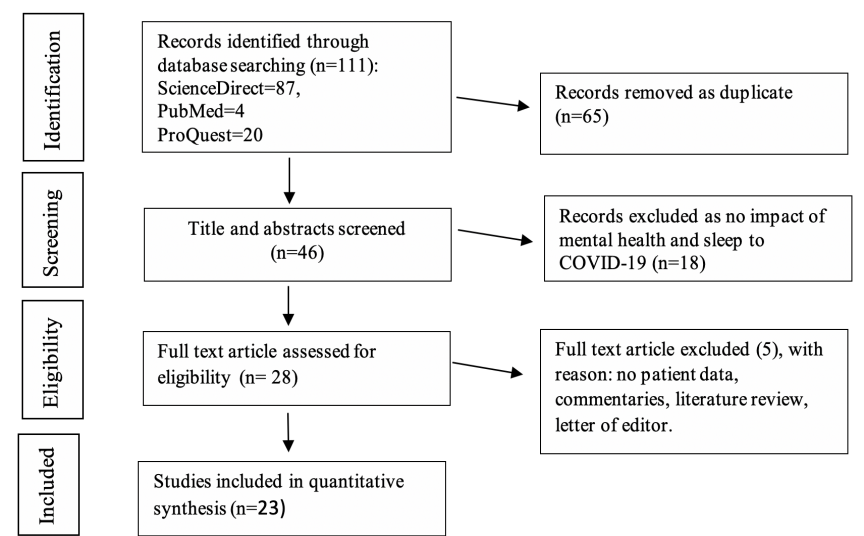

Figure 1. PRISMA 2009 Flow Diagram

search strategy in all the databases.

\section{Study Selection and Eligibility Criteria}

Two reviewers independently rated titles and abstracts obtained from electronic searches according to inclusion criteria to identify articles that could potentially qualify for the study. After removing articles that did not match the inclusion criteria, a complete article was obtained, then read by the same reviewers to determine which articles met the inclusion criteria requirements. However, if there was a dispute between the two reviewers, the third and fourth reviewer examined the article. The title and abstract of each relevant publication were screened. Fulltext articles were eligibility assessed after the initial screening.

The research criteria included in this study were: 1) followed a cross-sectional study design; 2) assessed the mental health status of the general public, medical workers, and non-medical workers during the COVID-19 pandemic; 3) assessed the sleep problems of the general public, medical workers, and non-medical workers during the COVID-19 pandemic; 4) used standardized and validated scales for measurement. The study was excluded if: 1) it was not written in English, 2) it did not have full-text availability.

\section{Data Extraction}

Relevant data taken from the study were extracted, including 1) main author and year of publication, 2) category, 3) country of the population studied, 4) study design, 5) sample size, 6) sample characteristics, 7) assessment tool, 8) prevalence, 9) summary.

\section{Quality Appraisal}

The Joanna Briggs Institute's (JBI) critical appraisal checklist tool for prevalence study was used to assess the quality of this study. ${ }^{14}$ The checklist consists of nine questions: the appropriate sample frame, sampling method justification, sample size justification, explanation of the study subjects and the setting, data analyzed using sufficient samples, statistical analysis suitability, reliable action for all participants, validity methods, and robust response rate. The checklist consists of four answer categories: yes, no, unclear, and not applicable. The answers for 
"yes" were given a score of 1 , and for "no," they were given a score of 0 . Total study quality scores ranged from $0-8$. In this review, the average score was above 5 , which means that the article was eligible for inclusion in the analysis.

\section{Results \\ Search Results}

This review revealed the impact of COVID-19 on mental health and sleep among the general public, medical workers, and non-medical workers. A total of 111 publications were identified. After the initial screening, 65 articles were removed due to duplication and leaving 46 articles. Eighteen articles were issued based on titles and abstracts. The remaining 28 full-text articles were assessed. Five articles were excluded because there were no patient data, commentaries, literature reviews, or editor letters. After the full-text screening, only 23 studies met the inclusion criteria.

\section{Study Characteristics}

The study characteristics and the main study findings of this study are summarized in Table 1 . There were 23 studies conducted in 11 different countries; China ( $\mathrm{n}=$ 13), Singapore and India $(n=1)$, Saudi Arabia $(n=1)$, India $(n=1)$, Turkey $(n=1)$, Iraq $(n=1)$, South Africa $(n=1)$, Bangladesh $(n=1)$, Hong Kong $(n=1)$, Algeria $(\mathrm{n}=1)$ and Australia $(\mathrm{n}=1)$. The majority of research was carried out on medical workers $(n=12)$ and the general public $(\mathrm{n}=10)$, with less carried out on with nonmedical workers $(n=1)$. The majority of studies had a cross-sectional study design $(n=22)$, and the rest was a survey $(n=1)$. Sample sizes ranged from 140 to 14,825 participants. The majority of study participants were over 18 years old. The main results selected in the studies were found to vary widely across studies. Sixteen studies found psychological problems, two found sleep problems, and six found psychological and sleep problems.

\section{The Impact of COVID-19 on Psychological Problems and Sleep}

The selected articles were analyzed one by one. Sixteen studies found psychological problems, two studies found sleep problems, and six studies found problems in psychology and sleep. ${ }^{7}$ This showed that COVID-19 has a real impact on psychological problems and sleep for the general public, medical workers, and nonmedical workers.

The Impact of COVID-19 on Psychological Problems and Sleep among Medical Workers

Medical workers suffered from psychological and sleep disorders. Psychological disorders included anxiety, psychological distress, depression, PTSD, somatic symptoms, high risk of mental illness, stress, and suicidal ideation. Of the total articles that reviewed psychological disorders experienced by medical workers, just under $50 \%$ of medical workers experienced severe anxiety, severe stress, and major depression. Thus, if this psychological disorder in medical workers is not handled properly, it will affect healthcare quality. Some of the research results below indicate psychology and sleep disturbances.

Medical workers experienced psychological disorders (anxiety and depression) and sleep disorders in two out of 12 studies. ${ }^{15}$ Psychological distress, anxiety, and depression were found in eight of the 12 articles studied. 16 Depressive symptoms and PTSD were found in a study conducted by Song et al. ${ }^{17}$ Medical workers also feel stress. ${ }^{18}$ Likewise, there was one study that reveals the existence of suicidal ideation. ${ }^{19}$

\section{The Impact of COVID-19 on Psychological and Sleep} Problems among Non-Medical Workers

The results showed that non-medical workers experienced psychological disorders, including depression. There were even participants who experienced significant depression, although the number was not large. In detail, the psychological disorders experienced by non-medical workers were as follows: $50.3 \%$ of participants reported clinically significant symptoms of depression. Among them, $33.0 \%$ of participants had mild depression, $10.5 \%$ of participants had moderate depression, $5.8 \%$ had moderate depression, and 1.0\% had severe depression. 3,20 The Impact of COVID-19 on Psychological and Sleep Problems on the General Public

The results of the selected article review showed that the general public also experienced psychological disturbances and sleep disorders due to COVID-19. Psychological disturbances make a person panic. The general public experienced sleep disturbances in two of the ten articles reviewed. ${ }^{21}$ The impact of COVID-19 on the disorder was found in two of ten articles. ${ }^{22}$ The general public experienced depression, anxiety, and stress in six out of ten articles reviewed. ${ }^{23}$

\section{Discussion}

This review explored medical workers' psychological and sleep status, non-medical workers, and the general public during the COVID-19 pandemic. In general, there was a higher prevalence of adverse psychology and sleep problems among medical workers, non-medical workers, and the general public. COVID-19 has a severe acute impact on psychological health and sleep quality for medical workers, non-medical workers, and the general public. The difference in the status of roles in society as medical workers, non-medical workers, and the general public affected the background causes of the problems that led to adverse psychology outcomes and sleep problems.

\section{The Impact of COVID-19 on Psychological Problems and} Sleep among Medical Workers

The COVID-19 pandemic had a severe psychological impact on medical workers and the general public, ${ }^{1}$ whe- 
Kesmas: Jurnal Kesehatan Masyarakat Nasional (National Public Health Journal). 2021; 16 (Special Issue 1): 65-74.

Table 1. Summary of Study Sample Characteristics, Study Design, Assessment Tools, Prevalence of Psychological and Sleep-Related Impact

\begin{tabular}{|c|c|c|c|c|c|c|c|c|}
\hline Author & Category & Country & $\begin{array}{l}\text { Study } \\
\text { Design }\end{array}$ & $\begin{array}{l}\text { Sample } \\
\text { Size (n) }\end{array}$ & $\begin{array}{l}\text { Sample } \\
\text { Characteristics }\end{array}$ & $\begin{array}{l}\text { Assessment } \\
\text { Tool }\end{array}$ & Prevalence & Summary \\
\hline $\begin{array}{l}\text { Wang, et al., } \\
2020^{24}\end{array}$ & $\begin{array}{l}\text { Medical } \\
\text { workers }\end{array}$ & China & $\begin{array}{l}\text { Cross- } \\
\text { sectional }\end{array}$ & $\begin{array}{l}274 \\
\text { medical } \\
\text { workers }\end{array}$ & Age $=33-40$ years & $\begin{array}{l}\text { GAD-7, } \\
\text { PHQ-9, } \\
\text { PSQI, } \\
\text { PSS-14, and } \\
\text { CD-RISC-1 }\end{array}$ & $\begin{array}{l}\text { Anxiety }=13.9 \% \\
\text { Depression }=16.1 \% \\
\text { Insomnia }=19.7 \%\end{array}$ & $\begin{array}{l}\text { The higher the probability and } \\
\text { intensity of being exposed to } \\
\text { COVID- } 19 \text { patients, the greater } \\
\text { the risk that medical staff will } \\
\text { suffer from mental disorders. }\end{array}$ \\
\hline $\begin{array}{l}\text { Song, et al., } \\
2020^{17}\end{array}$ & $\begin{array}{l}\text { Medical } \\
\text { workers }\end{array}$ & China & $\begin{array}{l}\text { Cross- } \\
\text { sectional }\end{array}$ & $\begin{array}{l}14,825 \\
\text { doctors } \\
\text { and nurses }\end{array}$ & The mean age $=34$ & $\begin{array}{l}\text { PSS, CES-D, } \\
\text { DSM-5 }\end{array}$ & $\begin{array}{l}\text { Depression symptoms } \\
=25.2 \% \\
\text { PTSD }=9.1 \%\end{array}$ & $\begin{array}{l}\text { Many of the medical staff in } \\
\text { the emergency department suf- } \\
\text { fer from symptoms of depres- } \\
\text { sion and PTSD. Nurses were } \\
\text { associated with a higher risk of } \\
\text { PTSD. }\end{array}$ \\
\hline
\end{tabular}

\begin{tabular}{|c|c|c|c|c|c|c|c|c|}
\hline $\begin{array}{l}\text { Juan. et al., } \\
2020^{16}\end{array}$ & $\begin{array}{l}\text { Medical } \\
\text { workers }\end{array}$ & China & $\begin{array}{l}\text { Cross- } \\
\text { sectional }\end{array}$ & $\begin{array}{l}456 \\
\text { doctors } \\
\text { and nurses }\end{array}$ & $\begin{array}{l}\text { The mean age }= \\
30.67 \pm 7.48 \text { years } \\
\text { (range: } 17-64 \text { years) }\end{array}$ & $\begin{array}{l}\text { IES-R, } \\
\text { GAD-7, } \\
\text { PHQ-9, } \\
\text { OCS, } \\
\text { PHQ-15 }\end{array}$ & $\begin{array}{l}\text { Psychological distress } \\
=37.5 \% \text {, Somatizati- } \\
\text { on symptoms }=33.3 \% \\
\text { Anxiety symptoms }= \\
31.6 \% \text {, Depression } \\
\text { symptoms }=29.6 \%\end{array}$ & $\begin{array}{l}\text { Negative psychological distress } \\
\text { on hospital staff. }\end{array}$ \\
\hline
\end{tabular}

\begin{tabular}{|c|c|c|c|c|c|c|c|c|}
\hline $\begin{array}{l}\text { Temsah, et al., } \\
2020^{25}\end{array}$ & $\begin{array}{l}\text { Medical } \\
\text { workers }\end{array}$ & $\begin{array}{l}\text { Saudi } \\
\text { Arabia }\end{array}$ & $\begin{array}{l}\text { Cross- } \\
\text { sectional }\end{array}$ & $\begin{array}{l}811 \text { healthcare } \\
\text { workers of a } \\
\text { tertiary care } \\
\text { teaching } \\
\text { hospital }\end{array}$ & Mean age $=36$ & GAD-7 & $\begin{array}{l}\text { Worried about COVID- } 19 \\
=41.1 \% \text {, Similarly worried } \\
\text { about both COVID-19 and } \\
\text { MERS-CoV= } 41.4 \% \text {, } \\
\text { Stressed about MERS-CoV } \\
=17.5 \%\end{array}$ & $\begin{array}{l}\text { COVID- } 19 \text { and MERS-CoV } \\
\text { pose significant levels of anxie- } \\
\text { ty and stress to healthcare wor- } \\
\text { kers caring for infected patients } \\
\text { especially at the risk of passing } \\
\text { the infection to their families. }\end{array}$ \\
\hline $\begin{array}{l}\text { Xiaoming, } \\
\text { et al., } 2020^{26}\end{array}$ & $\begin{array}{l}\text { Medical } \\
\text { workers }\end{array}$ & China & $\begin{array}{l}\text { Cross- } \\
\text { sectional }\end{array}$ & $\begin{array}{l}8,817 \text { hospital } \\
\text { workers }\end{array}$ & $\begin{array}{l}\text { The median age }= \\
=31\end{array}$ & $\begin{array}{l}\text { PHQ-9, } \\
\text { GAD-7 }\end{array}$ & $\begin{array}{l}\text { Depression }=30.2 \%, \\
\text { Anxiety }=20.7 \% \text {, Somatic } \\
\text { Symptoms }=46.2 \%, \\
\text { SSI }=6.5 \%\end{array}$ & $\begin{array}{l}\text { High level of psychological } \\
\text { impact and suicidal and self- } \\
\text { harm ideation on hospital staff. }\end{array}$ \\
\hline $\begin{array}{l}\text { Chew, et al., } \\
2020^{27}\end{array}$ & $\begin{array}{l}\text { Medical } \\
\text { workers }\end{array}$ & $\begin{array}{l}\text { Singapore } \\
\text { and } \\
\text { India }\end{array}$ & $\begin{array}{l}\text { Cross- } \\
\text { sectional }\end{array}$ & $\begin{array}{l}906 \text { healthcare } \\
\text { workers }\end{array}$ & $\begin{array}{l}\text { The median age }= \\
29 \text { (Interquartile } \\
\text { range: } 25-35 \text { ) years }\end{array}$ & $\begin{array}{l}\text { DASS-21, } \\
\text { IES-R }\end{array}$ & $\begin{array}{l}\text { Moderate to very severe } \\
\text { depression }=5.3 \% \text {, Mode- } \\
\text { rate to extremely severe } \\
\text { anxiety }=8.7 \% \text {, Moderate } \\
\text { to extremely severe stress } \\
=2.2 \% \text {, Moderate to severe } \\
\text { levels of psychological } \\
\text { distress }=3.8 \%\end{array}$ & $\begin{array}{l}\text { The prevalence of physical } \\
\text { symptoms was related to } \\
\text { psychological outcomes for } \\
\text { health workers during the } \\
\text { COVID-19 outbreak. }\end{array}$ \\
\hline $\begin{array}{l}\text { Hong, et al., } \\
2020^{19}\end{array}$ & $\begin{array}{l}\text { Medical } \\
\text { workers }\end{array}$ & China & $\begin{array}{l}\text { Cross- } \\
\text { sectional }\end{array}$ & 4,692 nurses & $>19$ years & $\begin{array}{l}\text { PHQ-9, } \\
\text { GAD-7, } \\
\text { PHQ-15 }\end{array}$ & $\begin{array}{l}\text { Depression symptoms }= \\
9.4 \%, \text { Represented anxiety } \\
=8.1 \% \text {, Somatic symptom } \\
=42.7 \% \text {, Suicidal ideation } \\
=6.5 \%\end{array}$ & $\begin{array}{l}\text { Overall the mental health of } \\
\text { frontline nurses during the } \\
\text { COVID-19 outbreak was } \\
\text { generally poor. }\end{array}$ \\
\hline $\begin{array}{l}\text { Zhu, et al., } \\
2020^{28}\end{array}$ & $\begin{array}{l}\text { Medical } \\
\text { workers }\end{array}$ & China & $\begin{array}{l}\text { Cross- } \\
\text { sectional }\end{array}$ & $\begin{array}{l}5,062 \text { health } \\
\text { workers }\end{array}$ & $>19$ years & $\begin{array}{l}\text { IES-R, } \\
\text { PHQ-9, } \\
\text { GAD-7 }\end{array}$ & $\begin{array}{l}\text { Stress }=29.8 \% \\
\text { Depression }=13.5 \% \\
\text { Anxiety symptoms }=24.1 \%\end{array}$ & $\begin{array}{l}\text { Health workers have a more } \\
\text { significant stigma against men- } \\
\text { tal problems than the general } \\
\text { public, so it was essential to } \\
\text { provide them with psychologi- } \\
\text { cal support. }\end{array}$ \\
\hline $\begin{array}{l}\text { Korkmaz, et } \\
\text { al., 202029 }\end{array}$ & Medical & Turkey & $\begin{array}{l}\text { Cross- } \\
\text { sectional }\end{array}$ & $\begin{array}{l}140 \text { healthcare } \\
\text { workers ( } 30 \\
\text { physicians, } 70 \\
\text { nurses, } 40 \text { assis- } \\
\text { tant healthcare) }\end{array}$ & $18-65$ years & $\begin{array}{l}\text { PSQI, PSI, } \\
\text { WHOQOL- } \\
\text { BREF, BAI }\end{array}$ & $\begin{array}{l}\text { Without anxiety }=29 \% \\
\text { Mild anxiety }=38 \% \\
\text { Moderate anxiety }=20 \% \\
\text { Severe anxiety }=13 \% \\
\text { Nurses PSQI and PSI scores, } \\
\text { statistically higher than } \\
\text { doctors and other staff }\end{array}$ & $\begin{array}{l}\text { Healthcare workers may experi } \\
\text { ence anxiety and sleep distur } \\
\text { bances that adversely affect } \\
\text { healthcare workers' problem- } \\
\text { solving skills and decrease their } \\
\text { quality of life. }\end{array}$ \\
\hline $\begin{array}{l}\text { Sahu, et al., } \\
2020^{18}\end{array}$ & $\begin{array}{l}\text { Medical } \\
\text { workers }\end{array}$ & India & Survey & $\begin{array}{l}611 \text { orthopedic } \\
\text { surgeons }\end{array}$ & $\begin{array}{l}<30 \text { years }- \text { more } \\
\text { than } 70 \text { years }\end{array}$ & $\begin{array}{l}\text { Questions } \\
\text { that were } \\
\text { shared with } \\
\text { orthopedic } \\
\text { surgeons }\end{array}$ & $\begin{array}{l}\text { Definitely stressed out }= \\
22.5 \%, \text { Mildly stressed } \\
\text { out }=40.5 \%\end{array}$ & $\begin{array}{l}\text { The psychological impact on } \\
\text { the orthopedic surgeon can be } \\
\text { a potential concern that } \\
\text { requires discussion through } \\
\text { open discussion. }\end{array}$ \\
\hline
\end{tabular}




\begin{tabular}{|c|c|c|c|c|c|c|c|c|}
\hline Author & Category & Country & $\begin{array}{l}\text { Study } \\
\text { Design }\end{array}$ & $\begin{array}{l}\text { Sample } \\
\text { Size }(n)\end{array}$ & $\begin{array}{l}\text { Sample } \\
\text { Characteristics }\end{array}$ & $\begin{array}{l}\text { Assessment } \\
\text { Tool }\end{array}$ & Prevalence & Summary \\
\hline $\begin{array}{l}\text { Abdullah \& } \\
\text { Musa., } 2020^{15}\end{array}$ & $\begin{array}{l}\text { Medical } \\
\text { workers }\end{array}$ & Iraq & $\begin{array}{l}\text { Cross- } \\
\text { sectional }\end{array}$ & $\begin{array}{l}268 \\
\text { physicians }\end{array}$ & $33-70$ years & $\begin{array}{l}\text { AIS, ICD-10 } \\
\text { The ladder } \\
\text { technique }(0-10)\end{array}$ & $\begin{array}{l}\text { Sleepless }=68.3 \% \\
\text { Stress }=93.7 \%\end{array}$ & $\begin{array}{l}\text { Doctors serving COVID-19 } \\
\text { patients have a negative effect on } \\
\text { sleep quality. }\end{array}$ \\
\hline $\begin{array}{l}\text { Fang, et al., } \\
202030\end{array}$ & $\begin{array}{l}\text { Non- } \\
\text { Medical } \\
\text { workers }\end{array}$ & China & $\begin{array}{l}\text { Cross- } \\
\text { sectional }\end{array}$ & $\begin{array}{l}191 \text { frontline } \\
\text { non-medical } \\
\text { workers }\end{array}$ & age $>20$ years & $\begin{array}{l}\text { PANAS, SRQ, } \\
\text { PHQ }\end{array}$ & $\begin{array}{l}\text { Symptoms of } \\
\text { depression }=50.3 \%\end{array}$ & $\begin{array}{l}\text { Frontline non-medical workers to } \\
\text { women and younger individuals } \\
\text { are more susceptible to depression. }\end{array}$ \\
\hline $\begin{array}{l}\text { Pillay, et } \\
\text { al., } 202021\end{array}$ & $\begin{array}{l}\text { General } \\
\text { public }\end{array}$ & $\begin{array}{l}\text { South } \\
\text { African }\end{array}$ & $\begin{array}{l}\text { Cross- } \\
\text { sectional }\end{array}$ & $\begin{array}{l}692 \text { elite and } \\
\text { semi-elite } \\
\text { athletes }\end{array}$ & $>18$ years & $\begin{array}{l}\text { Validated question- } \\
\text { naires on mainte- } \\
\text { nance of activity, } \\
\text { nutrition, and } \\
\text { mental state. }\end{array}$ & $\begin{array}{l}\text { Changed sleep } \\
\text { pattern }=79 \%, \\
\text { Depression }=52 \%\end{array}$ & $\begin{array}{l}\text { COVID-19 has psychological con- } \\
\text { sequences on athletes that can im- } \\
\text { pact safe returns to sport and } \\
\text { general health. }\end{array}$ \\
\hline $\begin{array}{l}\text { Wang, et al., } \\
2020^{7}\end{array}$ & $\begin{array}{l}\text { General } \\
\text { public }\end{array}$ & China & $\begin{array}{l}\text { Cross- } \\
\text { sectional }\end{array}$ & $\begin{array}{l}6,437 \\
\text { residents }\end{array}$ & $\begin{array}{l}\text { Mean age }= \\
31.4 \text { years }\end{array}$ & PSQI & $\begin{array}{l}\text { Sleep disturbances = } \\
17.65 \%\end{array}$ & $\begin{array}{l}\text { Almost one-fifth of residents had } \\
\text { sleep disorders }\end{array}$ \\
\hline $\begin{array}{l}\text { Huang \& } \\
\text { Zhao, } 2020^{31}\end{array}$ & $\begin{array}{l}\text { General } \\
\text { public }\end{array}$ & China & $\begin{array}{l}\text { Cross- } \\
\text { sectional }\end{array}$ & $\begin{array}{l}7,236 \text { Chinese } \\
\text { public }\end{array}$ & $6-80$ years & $\begin{array}{l}\text { PSQI, Chinese } \\
\text { version of GAD-7 } \\
\text { and CES-D }\end{array}$ & $\begin{array}{l}\text { Generalized anxiety } \\
\text { disorder }=35.1 \% \text {, } \\
\text { Depression symptoms } \\
=20.1 \%, \text { Worsened } \\
\text { sleep quality }=18.2 \%\end{array}$ & $\begin{array}{l}\text { Younger people spend too much } \\
\text { time thinking about the plague. }\end{array}$ \\
\hline
\end{tabular}

\begin{tabular}{|c|c|c|c|c|c|c|c|c|}
\hline $\begin{array}{l}\text { Duan, et al., } \\
2020^{23}\end{array}$ & $\begin{array}{l}\text { General } \\
\text { public }\end{array}$ & China & $\begin{array}{l}\text { Cross- } \\
\text { sectional }\end{array}$ & $\begin{array}{l}359 \text { children } \\
\text { and } 3254 \\
\text { adolescents }\end{array}$ & 7 - 18 years & $\begin{array}{l}\text { Spence Child Anxiety } \\
\text { Scale, Child Depres- } \\
\text { sion Inventory, and } \\
\text { Coping Style Scale }\end{array}$ & $\begin{array}{l}\text { Depression symptoms } \\
=22.28 \%\end{array}$ & $\begin{array}{l}\text { COVID-19 has a psychosocial } \\
\text { impact on children and adolescents }\end{array}$ \\
\hline
\end{tabular}

\begin{tabular}{|c|c|c|c|c|c|c|c|}
\hline $\begin{array}{l}\text { Ahmed, et al., } \\
2020^{32}\end{array}$ & $\begin{array}{l}\text { General } \\
\text { public }\end{array}$ & China & $\begin{array}{l}\text { Cross- } \\
\text { sectional }\end{array}$ & 1,074 people & $\begin{array}{l}14-68 \text { years } \\
(\mathrm{M}=33.54 \\
\text { years })\end{array}$ & $\begin{array}{l}\text { BAI, BDI-II, AUDIT } \\
\text { WEMWBS }\end{array}$ & $\begin{array}{l}\text { Anxiety }=\text { mild } 10.1 \% \text {, The psychological problems of } \\
\text { Moderate } 6.0 \% \text {, Severe society were interconnected in } \\
12.9 \% \text {, Depression }=\text { many ways related to COVID- } 19 \text {. } \\
\text { Mild } 10.2 \% \text {, Moderate } \\
17.8 \% \text {, Severe } 9.1 \% \text {, } \\
\text { Mental well-being = } \\
\text { lower } 32.1 \% \text {, average } \\
49.4 \% \text {, higher } 18.4 \%\end{array}$ \\
\hline
\end{tabular}

\begin{tabular}{|c|c|c|c|c|c|c|c|c|}
\hline $\begin{array}{l}\text { Islam, et al., } \\
2020^{33}\end{array}$ & $\begin{array}{l}\text { General } \\
\text { public }\end{array}$ & Bangladesh & $\begin{array}{l}\text { Cross- } \\
\text { sectional }\end{array}$ & $\begin{array}{l}1,311 \text { commu- } \\
\text { nity dwelling } \\
\text { individuals }\end{array}$ & $13-63$ years & GAD-7 & $\begin{array}{l}\text { Panic }=79.6 \%, \\
\text { Generalized anxiety }= \\
37.3 \%\end{array}$ & $\begin{array}{l}\text { Panic and generalized anxiety have } \\
\text { a large proportion in the general } \\
\text { population. }\end{array}$ \\
\hline $\begin{array}{l}\text { Ping, et al., } \\
2020^{34}\end{array}$ & $\begin{array}{l}\text { General } \\
\text { public }\end{array}$ & China & $\begin{array}{l}\text { Cross- } \\
\text { sectional }\end{array}$ & 1,139 people & $\begin{array}{l}\text { Mean age of } \\
38.3 \text { years } \\
\text { (12-78 years) }\end{array}$ & EQ-5D scale & $\begin{array}{l}\text { Anxiety/depression = } \\
17.6 \%\end{array}$ & $\begin{array}{l}\text { Anxiety/depression in people aged, } \\
\text { suffers of chronic diseases, worries } \\
\text { about contracting COVID-19, and } \\
\text { low income increases significantly. }\end{array}$ \\
\hline $\begin{array}{l}\text { Madani, et al., } \\
2020^{35}\end{array}$ & $\begin{array}{l}\text { General } \\
\text { public }\end{array}$ & Aljazair & $\begin{array}{l}\text { Cross- } \\
\text { sectional }\end{array}$ & 678 people & $14-74$ years & $\begin{array}{l}\text { Global questionnaire } \\
\text { measuring the impact } \\
\text { of confinement } \\
\text { during COVID-19 }\end{array}$ & $\begin{array}{l}\text { Anxiety }=50.3 \% \\
\text { Feels stressed }=48.2 \% \\
\text { Bad mood }=46.6 \% \\
\text { Do not stop thinking } \\
\text { about epidemic }=47.4 \%\end{array}$ & $\begin{array}{l}\text { Containment due to COVID- } 19 \\
\text { has raised anxiety in the general } \\
\text { public. }\end{array}$ \\
\hline $\begin{array}{l}\text { Newby, et al., } \\
2020^{36}\end{array}$ & $\begin{array}{l}\text { General } \\
\text { public }\end{array}$ & Australia & $\begin{array}{l}\text { Cross- } \\
\text { sectional }\end{array}$ & 5,070 adults & $18->75$ years & $\begin{array}{l}\text { DASS-21, The- } \\
\text { Whiteley-6 }\end{array}$ & $\begin{array}{l}\text { Depression }=62 \% \\
\text { Anxiety }=50 \% \\
\text { Stress }=64 \%\end{array}$ & $\begin{array}{l}\text { COVID- } 19 \text { has a severe acute im- } \\
\text { pact on people's mental health. }\end{array}$ \\
\hline $\begin{array}{l}\text { Wu et al., } \\
2020^{37}\end{array}$ & $\begin{array}{l}\text { Medical } \\
\text { workers }\end{array}$ & China & $\begin{array}{l}\text { Cross- } \\
\text { sectional }\end{array}$ & $\begin{array}{l}548 \text { medical } \\
\text { staff and me- } \\
\text { dical students }\end{array}$ & $\begin{array}{l}\text { Average age }= \\
28 \text { years }\end{array}$ & $\begin{array}{l}\text { The Kessler } 6 \text { Psycho- } \\
\text { logical Distress Scale }\end{array}$ & $\begin{array}{l}\text { High risk of severe } \\
\text { mental ilness }=37.23 \%\end{array}$ & $\begin{array}{l}\text { The medical team was suspectible } \\
\text { to severe mental illness. }\end{array}$ \\
\hline $\begin{array}{l}\text { Branda Yee- } \\
\text { Man Yu et al., } \\
2020^{22}\end{array}$ & $\begin{array}{l}\text { General } \\
\text { public }\end{array}$ & Hong Kong & $\begin{array}{l}\text { Cross- } \\
\text { sectional }\end{array}$ & $\begin{array}{l}1,338 \\
\text { young adults }\end{array}$ & $>18$ years & ISI & $\begin{array}{l}\text { Worsened sleep quality } \\
=38.3 \% \text {, Difficulty } \\
\text { sleep initiation }= \\
29.8 \% \text { Shortened } \\
\text { sleep duration }=29.1 \%\end{array}$ & $\begin{array}{l}\text { Most Hong Kong people feel that } \\
\text { their sleep has gotten worse since } \\
\text { the COVID- } 19 \text { outbreak due to a } \\
\text { lack of adequate masks. }\end{array}$ \\
\hline \multicolumn{9}{|c|}{$\begin{array}{l}\text { Note: Beck Depression Inventory-II (BDI-II), Beck Anxiety Inventory (BAI), Center for Epidemiologic Studies Depression Scale (CES-D), Connor-Davidson } \\
\text { Resilience Scale (CD-RISC)Depression, Anxiety, dan Stress Scale-21 item (DASS-21), Generalized Anxiety Disorder 7/2-item (GAD-7/2), Impact of Event Scale- } \\
\text { (Revised) (IES (-R), Kessler Psychological Distress Scale (K6 / 10), Insomnia Severity Index (ISI), Patient Health Questionnaire-9/2 (PHQ-9/15), Pittsburgh Sleep } \\
\text { Quality Index (PSQI), Problem Solving Inventory (PSI), Perceived Stress Scale (PSS), World Health Organization Quality of Life (WHOQOL), EuroQoL Quality of } \\
\text { Life Scale (EQ-5D) }\end{array}$} \\
\hline
\end{tabular}


re medical workers have a more significant stigma against mental problems than the general public. ${ }^{28}$ Medical workers who work on the frontline are vulnerable to psychological problems. Those who work in hospitals, respiratory departments, emergency departments, intensive care units, and infection departments are prone to anxiety, depression, PTSD, and negative psychological distress. ${ }^{17}$ The overall mental health of frontline nurses during the COVID-19 outbreak was generally poor. ${ }^{19}$ This happened because they are the personnel who directly handle COVID-19 patients at high risk of contracting the disease from their patients. These infected medical workers will have a high risk of transmitting the disease to their families. This situation becomes a burden for them; on the one hand, medical workers must help their patients while on the other hand, they must protect themselves and their families from the threat of COVID-19 transmitted from the patients they care.

Psychological problems can also occur due to the sharp increase in COVID-19 cases, which causes the workload of medical workers to increase as they try to take care of the patients. Meanwhile, the personal protective equipment (PPE) used to prevent medical workers from contracting the COVID-19 virus is inadequate for medical workers. ${ }^{10}$ The higher the probability and intensity of being exposed to COVID-19 patients, the greater the risk that medical workers will suffer from mental and sleep disorders. ${ }^{24}$ This means that workers who have a high risk of COVID-19 need attention, anticipation, and intervention to overcome the impact of COVID-19, namely mental and sleep disorders.

This study found that profession type and gender influenced the risk of psychological problems. Healthcare workers who are nurses and women are vulnerable to psychological stress. One review found that women and nurses have higher psychological distress than men and doctors. ${ }^{38}$ Health workers, especially nurses who work on the frontline, have the most contact with the most at risk of contracting COVID-19.

Similar to psychological problems, medical workers also experience vulnerability to sleep disorders. Medical workers who work on the frontline experience more sleep disturbances than non-medical professionals. ${ }^{39}$ An Iraqi study found that doctors serving COVID-19 patients experienced a negative effect on the quality of their sleep. ${ }^{15}$ Another study in China found that about $14 \%$ of doctors and nearly $16 \%$ of nurses showed symptoms of depression and insomnia. ${ }^{9}$ This may occur due to the increased mental and work pressures for medical workers. So that health workers are more at risk of exposure to psychological and sleep disorders.

Psychological and sleep problems experienced by medical workers can have a negative impact on their skills in solving healthcare workers' problems and may cause a decrease in their quality of life. ${ }^{29}$ Thus, their duties as professionals who work at the forefront of dealing with COVID-19 patients will undoubtedly be disrupted. A decrease in quality of life can make a person feel insignificant and leading to suicidal events. A high level of psychological impact can lead to suicide and self-harm ideation among medical workers. ${ }^{26}$ Given the high vulnerability of medical workers to psychological problems and sleep disorders, psychiatric intervention is needed to improve their mental health and sleep quality to resolve and terminate their problems.

\section{The Impact of COVID-19 on Psychological Problems and Sleep in Non-Medical Workers}

The impact of the psychological problems caused by the pandemic on non-medical workers is also increasing. They have the same risk of contracting the virus as medical workers, although not as much as the risk of transmission to medical workers. Medical workers and non-medical workers were subject to psychological stress during the pandemic. ${ }^{40}$ In this literature review study, only one study was found examining the impact of psychological problems and sleep due to pandemics on non-medical workers. However, the results of these studies can at least describe the problems they are experiencing during this pandemic.

The same principle applies to others: the younger the non-medical workers, the more susceptible they are to experiencing psychological problems. In terms of sex, female non-medical workers were more prone to experiencing psychological problems. A study in China showed that frontline non-medical workers, younger individuals, and women were more inclined to experiencing depression. ${ }^{30}$ However, there is still little research on the impact of psychology and sleep problems on non-medical workers during the pandemic. It may be necessary to perform more analysis in this field so that the proper intervention will be found to overcome the psychological problems they experience, considering that the profession of non-medical workers is also vital in dealing with problems caused by COVID-19.

The Impact of COVID-19 on Psychology and Sleep Problems among the General Public

In the general public, the various levels of outbreak severity, the stages of the outbreak in each region, the national economy, the government's readiness, the availability of medical facilities, and the dissemination of information related to COVID-19 can affect the psychological response of the community. ${ }^{3}$ Symptoms of psychological problems were more frequently seen at the start of an outbreak when individuals were required to undergo compulsory quarantine, sudden unemployment, and the uncertainty associated with the outbreak. ${ }^{41}$ Quarantine causes people who are usually free to carry out activities to become isolated to feel lonely in their li- 
ves. This can be a trigger for anxiety and depression. Social isolation and loneliness were closely linked to anxiety and depression. ${ }^{42}$ Job loss and uncertainty caused by the COVID-19 pandemic can be very stressful for a person. ${ }^{3}$ It left a feeling of shock and not ready to face the existing reality, resulting in panic and generalized anxiety. Containment due to COVID-19 has raised anxiety in the general public. ${ }^{35}$ In addition, the frequent exposure of a person to news related to COVID-19, misinformation, and fake news can cause symptoms of anxiety and stress. ${ }^{43}$

The impact of COVID-19 when viewed from an age perspective, the younger the age, the more susceptible to psychological effects that cause psychosocial problems. The findings were found in Duan et al.'s study, where COVID-19 has a psychosocial impact on children and adolescents. ${ }^{23}$ This was likely because most individuals under 40 were students who may experience more emotional distress. Usually, due to school closures, cancellation of social events, and lower learning efficiency only with online distance courses resulting in more homework assignments and postponement of exams. ${ }^{44}$ The pressures they experience can trigger PTSD and symptoms of depression. Several studies have found that student status is associated with higher levels of PTSD and depression symptoms during the COVID-19 outbreak than before. ${ }^{45}$ In addition, individuals who are less than 40 years old dwell on COVID-19 more. The younger population spends more time thinking about the pandemic, which impacts psychological problems and worsens sleep quality. ${ }^{31}$ Apart from young people, those with old age with chronic comorbidities are also susceptible to the psychological impact. Research on Chinese society has found that the level of anxiety/depression in aging people and people with chronic illnesses has increased significantly. ${ }^{34}$

Psychological problems and other problems experienced by the general public during the COVID-19 pandemic can cause sleep disturbances. The lack of availability of medical facilities as a preventive tool to protect the public from COVID-19 transmission has caused anxiety which has implications on sleep disturbances. For example, due to the lack of adequate masks, most Hong Kong people feel their sleep has worsened since the COVID-19 outbreak. ${ }^{16}$ Psychological problems and public sleep disorders are interconnected in many ways related to COVID-19.32 COVID-19 have become a nightmare for the society that afflicts the wider community. Research conducted on Chinese residents has found that almost onefifth of respondents had sleep disorders. ${ }^{7}$ Seeing the impact of psychological problems and sleep disorders on society, an adequate evidence-based intervention is required, accompanied by continuous and routine monitoring of the community's psychological consequences and sleep disorders.

A psychological response is actually needed when facing a problem, including a pandemic problem due to COVID-19. Acute psychological responses to stressful or traumatic events were sometimes protective and evolutionarily important, ${ }^{46}$ which will mobilize someone and force them to take precautions to protect themselves. ${ }^{3}$ However, psychological responses that have a bad impact must be avoided to worsen the problem. Follow-up studies after a pandemic are needed to assess the long-term psychological impact of the COVID-19 pandemic.

\section{Efforts to Reduce Symptoms of Psychological Disorders and Sleep Disorders}

The government needs to enforce strict policies on everything related to COVID-19 and explain procedures in an effort to alleviate the COVID-19 pandemic's psychological impact and the effects of sleep disturbance on medical workers, non-medical workers, and the general public. Attention and assistance should be prioritized for vulnerable groups such as females, those aged under 40 , the elderly with comorbidities, and those suffering from chronic diseases. The government must ensure that disseminating information related to COVID-19 has been conveyed accurately and transparently. The existence of validation from the government regarding news related to the pandemic was fundamental to avoid panic from incorrect information and update every time on information regarding preventive measures by health authorities to reassure people who fear infection. ${ }^{47}$ The government must also ensure that mental health services were easily accessible during the quarantine period, especially for those who desperately need psychological support. ${ }^{47}$ The government can also provide remote mental health services in online consultations and hotlines, ${ }^{48}$ due to quarantine. In addressing unemployment due to quarantine, the government can seek monetary support following the state budget and open new job opportunities for those who experience financial difficulties or lose their jobs during the pandemic. ${ }^{3}$

Apart from the government, individuals also have a fundamental role in helping to overcome psychological disorders and sleep disorders. Individuals can engage in positive activities that can relieve symptoms of psychological distress and their sleep disorders, such as regular exercise and maintaining a healthy diet. This has been shown to be effective in relieving and preventing symptoms of stress and depression. ${ }^{49}$ Individuals can also carry out distraction activities to divert attention from observing news related to COVID-19. This is done to avoid potential information that is not true. ${ }^{3}$ Distraction can be done by doing activities such as listening to songs, watching movies, painting, gardening, and cooking. If individuals want to get developments in information related to COVID-19, they must obtain it from news agencies 
and official organizations and only seek medical advice from trained healthcare professionals. ${ }^{3}$ Individuals must also continue to apply social distancing and health protocols.

\section{Strengths and Limitations}

The strength of this study is that it may be the first systematic review to examine and summarize the literature on psychological health and sleep disorders in medical workers, non-medical workers, and the general population during the COVID-19 outbreak. The weakness of this study is that most of the articles were obtained from Asian countries, so it is necessary to also disclose articles from European and American countries to determine the impact of COVID-19 on them.

\section{Conclusion}

This systematic review examines medical workers' psychological status and sleeps quality, non-medical workers, and the general public during the COVID-19 pandemic. A high prevalence of various psychological problems and sleep disorders is reported in most studies, both for medical workers, non-medical workers, and the general public. The COVID-19 pandemic represents an unprecedented threat to mental health and sleep needs in countries around the world. To overcome this problem, an intervention that focuses on preventing mental and sleep disorders is needed, and psychiatric interventions to improve mental health and sleep quality. Postpandemic cognitive and behavioral follow-up studies are required to assess the long-term psychological impact of the COVID-19 pandemic on medical workers, non-medical workers, and the general public. Also, ascertain whether training and support strategies can reduce their psychological morbidity and sleep disorders is also needed. Government policies are required to reduce the incidence of disease due to the COVID-19 virus, improving health facilities to protect themselves from contracting COVID-19, the need for continuous monitoring of the psychological consequences, and sleep disorders as a worldwide preparedness effort.

\begin{abstract}
Abbreviations
COVID-19: Coronavirus Disease 2019; WHO: World Health Organization; SARS: Severe Acute Respiratory Syndrome; PRISMA: Preferred Reporting Items for Systematic Reviews and Meta-Analysis; PTSD: Post-Traumatic Stress Disorder.
\end{abstract}

\section{Ethics Approval and Consent to Participate}

Not applicable.

\author{
Competing Interest \\ Not applicable.
}

\section{Availability of Data and Materials}

The data is publicly available from PubMed, ScienceDirect, ProQuest from 2019 to August 2020. The data of this study can be obtained from 23 eligible articles that have been included in the references.

\section{Authors' Contribution}

TS contributed to the overall design and article selection, conducted the data curation and formal analysis, wrote the original draft review, and prepared the manuscript. TS, CEK, HSM, and YH contributed to valida-tion, study quality appraisal, review, and editing of the draft. All other authors contributed to reviewing, editing, translating, and submission.

\section{Acknowledgment}

The authors would like to offer gratitude to Universitas Padjadjaran for providing the articles.

\section{References}

1. Luo M, Guo L, Yu M, Wang HJPr. The psychological and mental impact of coronavirus disease 2019 (COVID-19) on medical staff and general public-a systematic review and meta-analysis. 2020:113190.

2. Anand K, Karade S, Sen S, Gupta R. SARS-CoV-2: Camazotz's curse. Medical Journal, Armed Forces India. 2020;76(2):136.

3. Xiong J, Lipsitz O, Nasri F, Lui LM, Gill H, Phan L, et al. Impact of COVID-19 pandemic on mental health in the general population: a systematic review; 2020.

4. Munster VJ, Koopmans M, van Doremalen N, van Riel D, de Wit E. A novel coronavirus emerging in China-key questions for impact assessment. New England Journal of Medicine. 2020;382(8):692-4.

5. Cheng J, Huang C, Zhang G, Liu D, Li P, Lu C, et al. Epidemiological characteristics of novel coronavirus pneumonia in Henan. Chinese Journal of Tuberculosis and Respiratory Diseases. 2020;43:E027-E.

6. World Health Organization. Global research on coronavirus disease (COVID-19) 2020; 2020.

7. Wang J, Gong Y, Chen Z, Wu J, Feng J, Yan S, et al. Sleep disturbances among Chinese residents during the Coronavirus Disease 2019 outbreak and associated factors. 2020;74:199-203.

8. Thakur V, Jain AJB. COVID 2019-suicides: a global psychological pandemic. 2020;88:952.

9. Lai J, Ma S, Wang Y, Cai Z, Hu J, Wei N, et al. Factors associated with mental health outcomes among health care workers exposed to coronavirus disease 2019. 2020;3(3):e203976-e.

10. Xiang Y-T, Yang Y, Li W, Zhang L, Zhang Q, Cheung T, et al. Timely mental health care for the 2019 novel coronavirus outbreak is urgently needed. 2020;7(3):228-9.

11. Dhande KK, Sharma SJHF, Manufacturing Ei, Industries S. Influence of shift work in process industry on workers' occupational health, productivity, and family and social life: an ergonomic approach. 2011;21(3):260-8.

12. Patterson PD, Weaver MD, Frank RC, Warner CW, Martin-Gill C, Guyette FX, et al. Association between poor sleep, fatigue, and safety outcomes in emergency medical services providers. 2012;16(1):86-97.

13. Moher D, Liberati A, Tetzlaff J, Altman DG. Preferred reporting items for systematic reviews and meta-analyses: the PRISMA statement. Int J Surg. 2010;8(5):336-41.

14. Joanna Briggs Institute. Checklist for prevalence studies critical app- 
raisal tools for use in JBI Systematic reviews 2017; 2020.

15. Abdulah DM, Musa DHJSMX. Insomnia and stress of physicians during COVID-19 outbreak. 2020;2:100017.

16. Juan Y, Yuanyuan C, Qiuxiang Y, Cong L, Xiaofeng L, Yundong Z, et al. Psychological distress surveillance and related impact analysis of hospital staff during the COVID-19 epidemic in Chongqing, China. 2020;103:152198.

17. Song X, Fu W, Liu X, Luo Z, Wang R, Zhou N, et al. Mental health status of medical staff in emergency departments during the Coronavirus disease 2019 epidemic in China. 2020;88:60-5.

18. Sahu D, Agrawal T, Rathod V, Bagaria V. Impact of COVID 19 lockdown on orthopaedic surgeons in India: a survey. 2020;11:S283-S90.

19. Hong S, Ai M, Xu X, Wang W, Chen J, Zhang Q, et al. Immediate psychological impact on nurses working at 42 government-designated hospitals during COVID-19 outbreak in China: a cross-sectional study. 2021;69(1):6-12.

20. McIntyre RS, Lee Y. Projected increases in suicide in Canada as a consequence of COVID-19. J Psychiatry research. 2020;290:113104.

21. Pillay L, van Rensburg DCCJ, van Rensburg AJ, Ramagole DA, Holtzhausen L, Dijkstra HP, et al. Nowhere to hide: the significant impact of coronavirus disease 2019 (COVID-19) measures on elite and semi-elite South African athletes. 2020;23(7):670-9.

22. Yu BY-M, Yeung W-F, Lam JC-S, Yuen SC-S, Lam SC, Chung VC-H, et al. prevalence of sleep disturbances during COVID-19 outbreak in an urban Chinese population: a cross-sectional study. 2020;74:18-24.

23. Duan L, Shao X, Wang Y, Huang Y, Miao J, Yang X, et al. An investigation of mental health status of children and adolescents in china during the outbreak of COVID-19. 2020;275:112-8.

24. Wang L-Q, Zhang M, Liu G-M, Nan S-Y, Li T, Xu L, et al. Psychological impact of coronavirus disease (2019) (COVID-19) epidemic on medical staff in different posts in China: a multicenter study. Journal of Psychiatric Research. 2020;129:198-205.

25. Temsah M-H, Al-Sohime F, Alamro N, Al-Eyadhy A, Al-Hasan K, Jamal A, et al. The psychological impact of COVID-19 pandemic on health care workers in a MERS-CoV endemic country. Journal of Infection and Public Health. 2020;13(6):877-82.

26. Su H, Wo W, Jianmei C, Qi Z, et al. The psychological status of 8817 hospital workers during COVID-19 epidemic: a cross-sectional study in Chongqing. 2020;276:555-61.

27. Chew NW, Lee GK, Tan BY, Jing M, Goh Y, Ngiam NJ, et al. A multinational, multicentre study on the psychological outcomes and associated physical symptoms amongst healthcare workers during COVID19 outbreak. 2020;88:559-65.

28. Zhu Z, Xu S, Wang H, Liu Z, Wu J, Li G, et al. COVID-19 in Wuhan: sociodemographic characteristics and hospital support measures associated with the immediate psychological impact on healthcare workers. 2020;24:100443.

29. Korkmaz S, Kazgan A, Çekiç S, Tartar AS, Balcı HN, Atmaca M. The anxiety levels, quality of sleep and life and problem-solving skills in healthcare workers employed in COVID-19 services. Journal of Clinical Neuroscience. 2020;80:131-6.

30. Fang X, Zhang J, Teng C, Zhao K, Su K-P, Wang Z, et al. Depressive symptoms in the frontline non-medical workers during the COVID-19 outbreak in Wuhan. Journal of Affective Disorders. 2020;276:441-5.
31. Huang Y, Zhao N. Generalized anxiety disorder, depressive symptoms and sleep quality during COVID-19 outbreak in China: a web-based cross-sectional survey. J Psychiatry research. 2020;288:112954.

32. Ahmed MZ, Ahmed O, Aibao Z, Hanbin S, Siyu L, Ahmad A. Epidemic of COVID-19 in China and associated psychological problems. Asian Journal of Psychiatry. 2020;51:102092.

33. Islam MS, Ferdous MZ, Potenza MN. Panic and generalized anxiety during the COVID-19 pandemic among Bangladeshi people: an online pilot survey early in the outbreak. Journal of Affective Disorders. 2020;276:30-7.

34. Ping W, Zheng J, Niu X, Guo C, Zhang J, Yang H, et al. Evaluation of health-related quality of life using EQ-5D in China during the COVID19 pandemic. 2020;15(6):e0234850.

35. Madani A, Boutebal SE, Bryant CR. The psychological impact of confinement linked to the coronavirus epidemic COVID-19 in Algeria. International Journal of Environmental Research and Public Health. 2020;17(10):3604.

36. Newby JM, O’Moore K, Tang S, Christensen H, Faasse KJPo. Acute mental health responses during the COVID-19 pandemic in Australia. 2020;15(7):e0236562.

37. Wu S, Li Z, Li Z, Xiang W, Yuan Y, Liu Y, et al. The mental state and risk factors of Chinese medical staff and medical students in early stages of the COVID-19 epidemic. Comprehensive Psychiatry. 2020:152202.

38. Qiu J, Shen B, Zhao M, Wang Z, Xie B, Xu YJGp. A nationwide survey of psychological distress among Chinese people in the COVID-19 epidemic: implications and policy recommendations. 2020;33(2).

39. Herrero San Martin A, Parra Serrano J, Diaz Cambriles T, Arias Arias EM, Muñoz Méndez J, del Yerro Álvarez MJ, et al. Sleep characteristics in health workers exposed to the COVID-19 pandemic. Sleep Medicine; 2020.

40. Sheraton M, Deo N, Dutt T, Surani S, Hall-Flavin D, Kashyap R. Psychological effects of the COVID 19 pandemic on healthcare workers globally: a systematic review. Psychiatry Research. 2020;292:113360.

41. Pan A, Liu L, Wang C, Guo H, Hao X, Wang Q, et al. Association of public health interventions with the epidemiology of the COVID-19 outbreak in Wuhan, China. 2020;323(19):1915-23.

42. Holmes EA, O’Connor RC, Perry VH, Tracey I, Wessely S, Arseneault L, et al. Multidisciplinary research priorities for the COVID-19 pandemic: a call for action for mental health science; 2020.

43. Gao J, Zheng P, Jia Y, Chen H, Mao Y, Chen S, et al. Mental health problems and social media exposure during COVID-19 outbreak. 2020;15(4):e0231924.

44. Cao W, Fang Z, Hou G, Han M, Xu X, Dong J, et al. The psychological impact of the COVID-19 epidemic on college students in China. Psychiatry research. 2020:112934.

45. Lei L, Huang X, Zhang S, Yang J, Yang L, Xu MJMsmimjoe, et al. Comparison of prevalence and associated factors of anxiety and depression among people affected by versus people unaffected by quarantine during the COVID-19 epidemic in Southwestern China. 2020;26:e924609-1.

46. Yaribeygi H, Panahi Y, Sahraei H, Johnston TP, Sahebkar AJEj. The impact of stress on body function: A review. 2017;16:1057. 
Kesmas: Jurnal Kesehatan Masyarakat Nasional (National Public Health Journal). 2021; 16 (Special Issue 1): 65-74.

47. Tran BX, Dang AK, Thai PK, Le HT, Le XTT, Do TTT, et al. Coverage of health information by different sources in communities: implication for COVID-19 epidemic response. 2020;17(10):3577.

48. Liu S, Yang L, Zhang C, Xiang Y-T, Liu Z, Hu S, et al. Online mental health services in China during the COVID-19 outbreak. 2020;7(4):e17-e8.

49. Carek PJ, Laibstain SE, Carek SM. Exercise for the treatment of depression and anxiety. Int J Psychiatry Med. 2011;41(1):15-28. 